\title{
Star formation environments and the distribution of binary separationst
}

\author{
Wolfgang Brandner ${ }^{2,3}$, Rainer Köhler ${ }^{4,5}$ \\ ${ }^{2}$ Caltech - JPL/IPAC, Mail Code 100-22, Pasadena, CA 91125, USA \\ ${ }^{3}$ University of Illinois at Urbana-Champaign, Department of Astronomy, 1002 West Green Street, Urbana, IL \\ 61801, USA \\ ${ }^{4}$ Max-Planck-Institut für Astronomie, Königstuhl 17, D-69117 Heidelberg, Germany \\ ${ }^{5}$ Astrophysikalisches Institut Potsdam, An der Sternwarte 16, D-14482 Potsdam, Germany
}

\begin{abstract}
We have carried out K-band speckle observations of a sample of 114 X-ray selected weak-line T Tauri stars in the nearby Scorpius-Centaurus OB association. We find that for binary T Tauri stars closely associated to the early type stars in Upper Scorpius, the youngest subgroup of the OB association, the peak in the distribution of binary separations is at 90 A.U. For binary T Tauri stars located in the direction of an older subgroup, but not closely associated to early type stars, the peak in the distribution is at 215 A.U. A Kolmogorov-Smirnov test indicates that the two binary populations do not result from the same distribution at a significance level of $98 \%$.

Apparently, the same physical conditions which facilitate the formation of massive stars also facilitate the formation of closer binaries among low-mass stars, whereas physical conditions unfavorable for the formation of massive stars lead to the formation of wider binaries among low-mass stars. The outcome of the binary formation process might be related to the internal turbulence and the angular momentum of molecular cloud cores, magnetic field, the initial temperature within a cloud, or - most likely - a combination of all of these.

We conclude that the distribution of binary separations is not a universal quantity, and that the broad distribution of binary separations observed among main-sequence stars can be explained by a superposition of more peaked binary distributions resulting from various star forming environments. The overall binary frequency among pre-mainsequence stars in individual star forming regions is not necessarily higher than among main-sequence stars.
\end{abstract}

Subject headings: open clusters and associations: individual (Scorpius-Centaurus) binaries: visual — stars: formation — stars: pre-main sequence

${ }^{1}$ Based on observations obtained at the European Southern Observatory, La Silla; ESO Proposal No. 53.7-0121, 55.E-0968, 56.MPI-8 


\section{Introduction}

Taurus-Auriga is the star forming region which has been most thoroughly surveyed for pre-main-sequence binary and multiple systems (see Mathieu 1994 for a review). For separations from 15 A.U. to 2000 A.U., the binary frequency among T Tauri stars in Taurus is 1.9 times as high as among nearby main-sequence stars (Köhler \& Leinert 1998). Extrapolating over the whole range of separations yields a binary frequency of $100 \%$, i. e., each T Tauri star in Taurus should be member of a binary or multiple systems. This apparent overabundance of binaries among pre-mainsequence stars is puzzling. One possible explanation is a decrease in the binary frequency as a function of the age of a stellar population (Patience et al. 1998). However, a T association like Taurus might not be the typical birthplace for low-mass stars, as up to $80 \%$ of all low-mass stars could originate in OB associations (Miller \& Scalo 1978; see also Zinnecker et al. 1992).

Scorpius-Centaurus is the most nearby OB association at a distance of about 145 parsec (de Zeeuw et al. 1998). It consists of three subgroups (cf. Figure 1) with ages ranging from 5 to $13 \mathrm{Myr}$ (de Geus et al. 1989). Upper Centaurus-Lupus (UCL) is the oldest subgroup of the association. Star formation started here $13 \mathrm{Myr}$ ago and subsequently progressed throughout the parental giant molecular cloud (e.g. Blaauw 1991 and references therein).

Based on observations with the EINSTEIN X-ray satellite, Walter et al. (1994) identified 28 weak-line T Tauri stars (WTTS) in Upper Scorpius (US), the youngest subgroup. 10 of these have been surveyed by Ghez et al. (1993) for binary or multiple systems, and three binaries have been detected. The EINSTEIN fields covered only a small fraction of US (Fig. 2) and the 28 WTTS did not allow for a statistical meaningful study of binary frequencies and separations. A search for visual binary stars among 74 ROSAT selected WTTS and post-T Tauri stars in US (Kunkel et al., in prep) was carried by Brandner et al. (1996). This survey was sensitive to binary separations down to $0 . \prime 8$, and revealed a rather high binary frequency in the region located between US and UCL ('US-B') and an apparent absence of wide visual binary stars in the center of US ('US-A').

In order to identify closer binary systems and to get a better statistics on possible spatial variations of binary star properties, we have carried out a speckle survey of 114 WTTS in US based on the lists by Wal- ter et al. (1994) and Kunkel et al. (in prep).

\section{Observations and Data Analysis}

The speckle data were obtained with the SHARPcamera at the ESO 3.5m New Technology Telescope on La Silla, Chile, in May 1994 and July 1995. All observations were performed in the K-band. In order to estimate the local density of background sources, we obtained additional infrared images with the ESO/MPIA $2.2 \mathrm{~m}$ telescope on La Silla in March 1996 using the IRAC2b camera. The detailed results, i. e., separations, position angles, photometry of the individual binary components, and de-biased binary frequencies will be presented in a forthcoming paper (Köhler et al., in prep).

Here we concentrate on the distribution of the binary separations. In total we observed 68 WTTS in US-A (26 from the list by Walter et al. 1994, and 42 new WTTS from Kunkel et al., in prep) and 46 WTTS in US-B (Kunkel et al., in prep). As US is projected onto the Galactic bulge and in the Kband has a $6 \times$ higher density of background sources than Taurus (Köhler 1997; Köhler et al., in prep.), we used a separation of $3^{\prime \prime}$ as the upper cut-off, in order to reduce contamination by background giants. The distribution of brightness ratios as a function of binary separations is rather uniform for separations between $0^{\prime \prime} 1$ and $3^{\prime \prime}$. The limiting magnitude for undetected companions has a $1 / \mathrm{d}$ dependence with a typical brightness ratio limit of 0.16 at 0.5 and 0.1 at 0 .' 8 . Among the binaries with separations between $0{ }^{\prime \prime} 8$ and $3^{\prime \prime}$, only two have brightness ratios $\leq 0.1$. For separations $\geq 4^{\prime \prime}$ we find a larger number of visual binaries with brightness ratios $\leq 0.1$, most of which are very likely chance projections. Counting binaries with separations between $0^{\prime \prime} 1$ and $3^{\prime \prime}$, we detect 21 binaries in US-A and 18 binaries (if we count one hierarchical triple system as two binaries) in US-B.

\section{Comparison of US-A and US-B}

The age of the WTTS population in US is under debate. Walter et al. (1994) derive ages of 1-2 Myr for the WTTS and conclude that the WTTS formed 3-4 Myr after the early-type stars in US. Kunkel et al. (in prep) and Martín (1998) find that the age of WTTS in Upper Scorpius is in agreement with the age of the B stars. Our speckle survey uncovers that almost all of the brighter M-type WTTS in US-A and US-B are in fact binaries. Only if they are not recognized as 


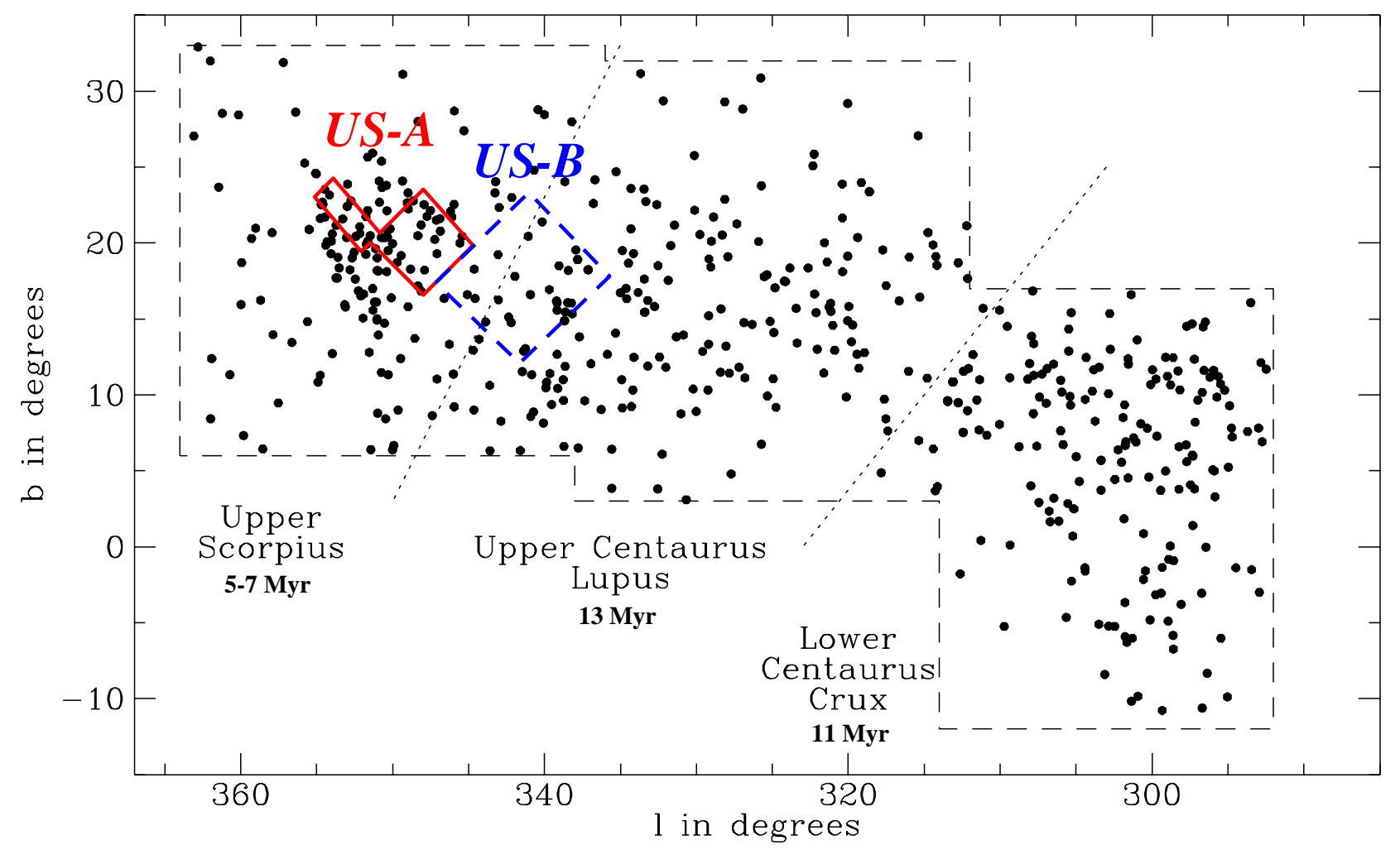

Fig. 1.- The spatial distribution of 532 proper motion members of the Scorpius-Centaurus association based on HIPPARCOS measurements (adapted from de Zeeuw et al. 1998). The boundaries between the subgroups Upper Scorpius (US), Upper Centaurus Lupus (UCL), and Lower Centaurus Crux (LCC) are indicated by dotted lines. Star formation has progressed from the oldest subgroup UCL towards the younger subgroups US and LCC. The two adjacent fields of our survey for binary T Tauri stars, centered on US (US-A, solid lines) and at the interface between US and UCL (US-B, dashed lines) are outlined.

such, they are erroneously placed too high in the H-R diagram (cf. Simon et al. 1993, Brandner \& Zinnecker 1997, Ghez et al. 1997) and an age of $\leq 1 \mathrm{Myr}$ is derived.

The WTTS in US-A and US-B show the same mix in spectral types and ages. The average Lithium abundance is slightly lower in US-B compared to USA. If this is not due to age, then it might be related to the difference in binary separations. Wider binaries sustain massive circumstellar disks longer than closer binaries. Without a massive circumstellar disk a T Tauri star cannot effectively dissipate angular momentum and thus spins up while contracting (e.g. Bodenheimer 1995). Faster rotation should enhance the dynamo effect and the resulting stronger inter- nal magnetic fields could in turn aggravate convection and thus slow down the depletion of Lithium on the surface.

\section{The Distribution of Binary Separations}

Based on counts of background sources, we expect two of the 39 binaries in our sample to be pure chance projections. A $5 \%$ contamination by bogus binaries should not strongly affect our analysis. Without correction for selection effects, the 'raw' binary frequency in US-B $(39 \% \pm 9 \%)$ is somewhat higher than in USA $(31 \% \pm 7 \%)$, but the difference is not statistically significant.

The distribution of binary separations, however, is clearly distinct in the two regions: about $80 \%$ of the 

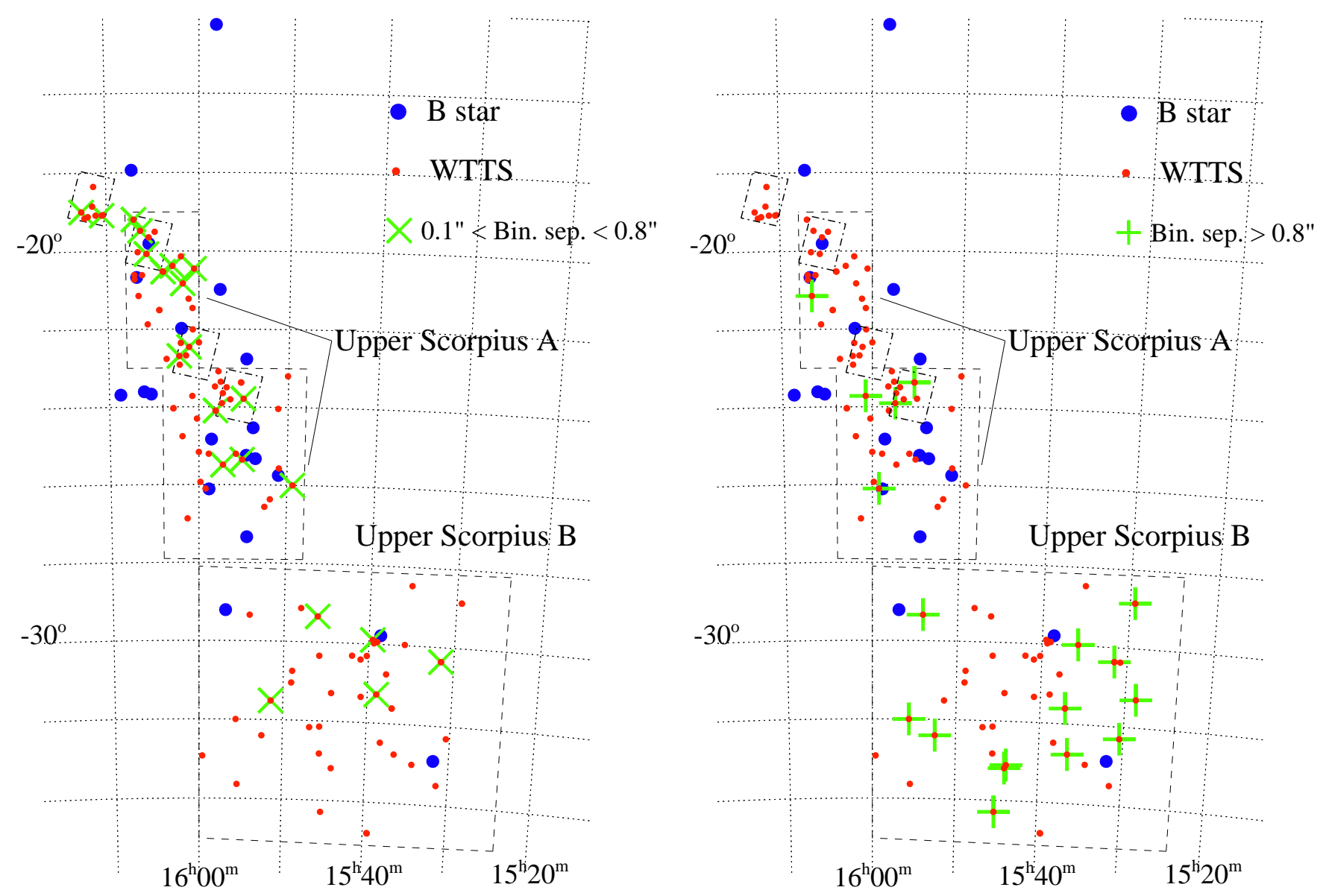

Fig. 2.- Close-up of Upper Scorpius A and B. The regions designated as Upper Scorpius A and B and studied as ROSAT follow-ups are outlined by dashed lines, the regions observed by the EINSTEIN satellite are outlined by dash-dotted lines. The closer binary WTTS cluster in Upper Scorpius A, whereas the wider binaries are more frequent in Upper Scorpius B.

binary systems in US-A have separations less than $0 . \prime 8$, whereas in US-B $70 \%$ of the binary systems have separations greater than $0 . \prime 8$ (Fig. 2). The average separation of binary systems in US-B is 1 ". 47 , more than twice as large as the average separation of binary systems in US-A (0!'63).

In order to transform angular to physical separations, we need to know the distance to our stars. The average distance to Scorpius-Centaurus, as derived from HIPPARCOS measurements of early type stars, is 145 parsec for US and 140 parsec for UCL (de Zeeuw et al. 1998). For four of the WTTS in US-B HIPPARCOS derived meaningful distances (Neuhäuser \& Brandner 1998; Wichmann et al. 1998). One of these stars (HD 140637) is clearly a foreground star at a distance of $41_{-2}^{+2}$ parsec. The three other stars (all of them are binaries) have HIPPARCOS distances be- tween 90 parsec and 160 parsec, albeit with rather large uncertainties ( \pm 20 to \pm 80 parsec). We see no evidence that the WTTS in US-B are more than a factor of 2 closer than the WTTS in US-A as would be necessary in order to explain the difference in the distribution of angular binary separations by the distance. In the following we assume that the WTTS in US-A and US-B are at the same distance as the early type stars in Scorpius-Centaurus. Figure 3 shows a histogram of the distribution of binary separations in US-A and in US-B. While the distribution peaks around 90 A.U. in US-A, the peak of the distribution is clearly shifted towards larger separations $(\approx 215$ A.U.) in US-B. A Kolmogorov-Smirnov test indicates that both binary populations are drawn from different distributions at a significance level of $98 \%$. Removing the two widest binaries in US-B from the sample (assuming that they might be chance projections) lowers 

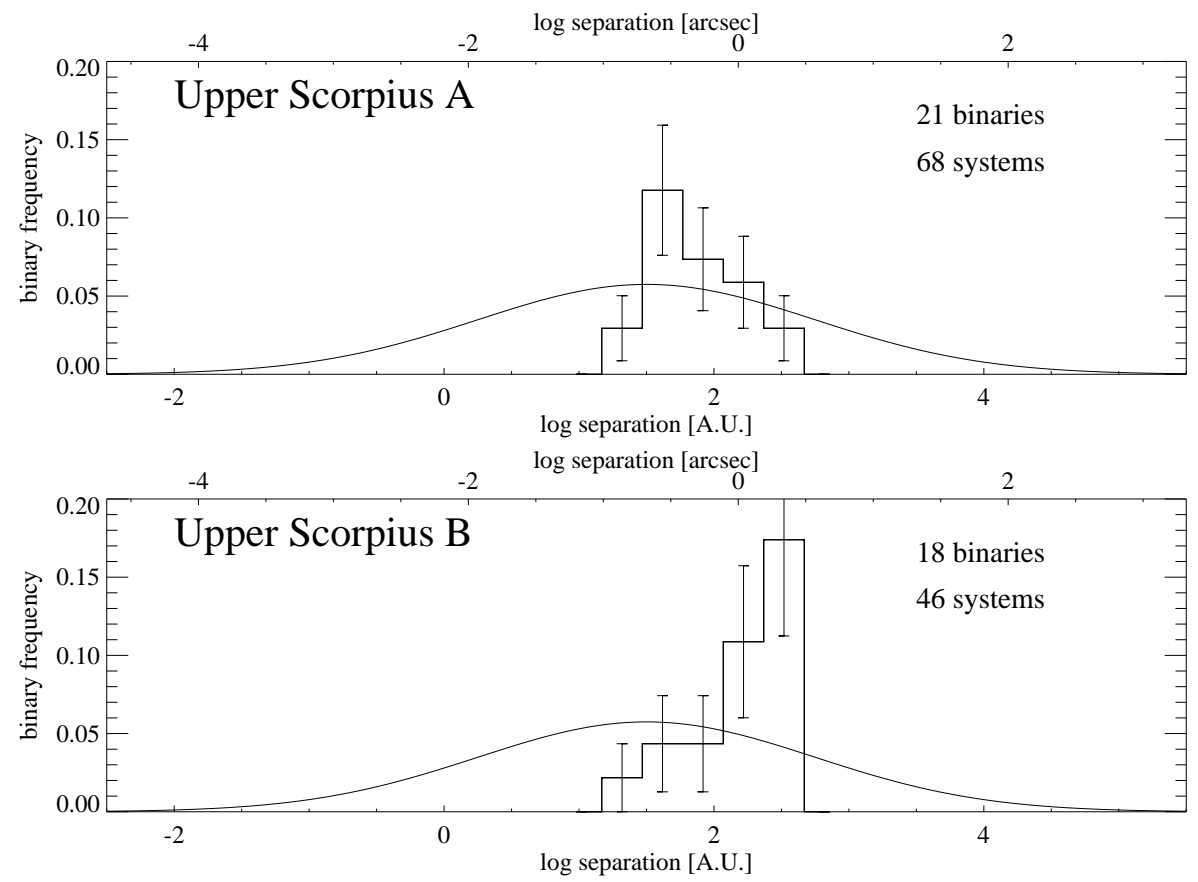

Fig. 3.- Histograms of the distribution of binary star separations for weak-line T Tauri stars in Upper Scorpius A and B. The log-normal type distribution found by Duquennoy \& Mayor (1991) for main-sequence stars is shown as well.

the significance level to $\% 96$.

\section{Star forming environments and Binaries}

In Scorpius-Centaurus the molecular cloud in which the star formation originated has long been dispersed by ionizing radiation and fast stellar winds from the massive stars and by supernova explosions. Today, the Lupus dark clouds to the south and the $\rho$ Ophiuchi dark clouds to the east of US are the only sites of ongoing (low-mass) star formation in the outskirts of a once giant molecular cloud. No direct trace of the original physical conditions remains. We can, however, deduce that the same environment which facilitated the formation of massive stars in US-A also facilitated the formation of closer binaries among the low-mass stars, whereas in US-B the original physical conditions were such that almost no massive stars formed, and preferentially wide binaries among the low-mass stars.

Other studies might reveal a similar trend. In their search for visual binaries in nearby dark clouds Reipurth \& Zinnecker (1993) found a weak anticorrelation between the binary frequency and the number of young stars in the clouds. This could be due to a shift in the peak of the distribution of binary separations, with the larger clouds producing preferentially closer binaries than the smaller clouds. Padgett et al. (1997) and Petr et al. (1998) studied the binary frequency in Orion (distance $\approx 460 \mathrm{pc}$ ) and found a deficit of "wide" ( $\geq 0$ '! 1 , i. e., 50 A.U.) premain-sequence binaries among T Tauri stars closely associated to the high-mass stars in the Trapezium cluster.

Binary stars form due to fragmentation during collapse (e.g. Boss 1997 and references therein). The binary separation depends on the initial angular momentum and on the critical density at which the magnetic field decouples from the matter (Mouschovias 1977). The critical density $n_{\text {cr }}$ relates to the separation $\mathrm{a}_{\mathrm{b}}$ of a binary systems as $n_{\mathrm{cr}} \propto \mathrm{a}_{\mathrm{b}}^{-3 / 4}$. Part of the initial angular momentum of a protobinary might be transported outwards by means of a circumbinary disk or might be dissipated due to the gravitational interaction of the circumstellar disks of the individual components. The temperature structure of a molecular cloud could influence the number of binary stars (Durisen \& Sterzik 1994) as well as their typical separation (Durisen et al., in prep.). The limited parameter space due to a higher ambient cloud temperature would preferably lead to the formation of closer binaries.

Molecular clouds in the process of forming (binary) stars have to be studied in order to learn which physical quantities determine the outcome of the binary formation process. 


\section{Conclusion}

We have shown that the distributions of binary separations among weak-line T Tauri stars in two adjacent fields ('US-A' and 'US-B') in the ScorpiusCentaurus OB association are clearly distinct from each other and considerably more peaked than the (broad) distribution of binary separations observed among main-sequence field stars. In US-A, the WTTS are closely associated with B type stars, whereas in US-B only a few early type stars are present. We conclude that the same physical conditions which facilitate the formation of massive stars also facilitate the formation of closer binaries among low-mass stars, whereas physical conditions unfavorable for the formation of massive stars lead to the formation of wider binaries among low-mass stars.

The outcome of the binary formation process might be determined by the critical density at which the magnetic field support breaks down, the internal turbulence and the angular momentum of molecular cloud cores, the initial temperature within a cloud, or - most likely - a combination of all of these.

We further conclude that the distribution of binary separations is not a universal quantity. Instead, both the peak and the width of the distribution might vary from one star forming region to the next. The broad distribution of binary separations observed among main-sequence field stars can be understood as a superposition of binary populations originating in various star forming environments with very distinct peaks in the distribution of binary separations.

The apparent overabundance of binaries among $\mathrm{T}$ Tauri stars in the Taurus-Auriga $\mathrm{T}$ association might be explained by the fact that the distribution of binary separations there is strongly peaked towards $\approx 30$ A.U. Extrapolating from this very pronounced peak over the whole range of possible binary separations then leads to an erroneously high estimate of the overall binary frequency.

We would like to thank Michael Kunkel for making his list of WTTS in US available to us prior to publication. We acknowledge inspiring discussions with Dick Durisen, Christoph Leinert, Michael Sterzik, and Hans Zinnecker, who initiated this project. WB acknowledges support under NASA/HST grant GO7412.01-94A.

\section{REFERENCES}

Blaauw, A. 1991 OB Associations and the Fossil Record of Star Formation, in The Physics of Star Formation and Early Stellar Evolution, eds. C.J. Lada \& N.D. Kylafis, p. 125

Bodenheimer, P. 1995, ARA\&A 33, 199

Boss, A.P. 1997, ApJ 483, 309

Brandner, W., Alcalá, J.M., Kunkel, M., Moneti, A., Zinnecker, H. 1996, A\&A 307, 121

Brandner, W., Zinnecker, H. 1997, A\&A 321, 220

Duquennoy, A., Mayor, M. 1991, A\&A 248, 485

Durisen, R.H., Sterzik, M.F. 1994, A\&A 286, 84

de Geus, E.J., de Zeeuw, P.T., Lub, J. 1989, A\&A 216,44

Ghez, A.M., Neugebauer, G., Matthews, K. 1993, AJ 106,2005

Ghez, A.M., White, R.J., Simon, M. 1997, ApJ 490, 353

Köhler, R. 1997, PhD thesis, Universität Heidelberg

Köhler, R., Leinert, Ch. 1998, A\&A 331, 977

Martín, E.L. 1998, AJ 115, 351

Mathieu, R.D. 1994, ARA\&A 32, 465

Miller, G.E., Scalo J.M. 1978, PASP 90, 506

Mouschovias, T.Ch. 1977, ApJ 211, 147

Neuhäuser, R., Brandner, W. 1998, A\&A 330, L29

Padgett, D.L., Strom, S.E., Ghez, A.M. 1997, ApJ 477,705

Patience, J., Ghez, A.M., Reid, I.N., Weinberger, A.J., Matthews, K. 1998, AJ, in press (May issue)

Petr, M.G., Coudé du Foresto, V., Beckwith, S.V.W., Richichi, A., McCaughrean, M.J. 1998, ApJ 499, in press (Jul 20 issue)

Reipurth, B., Zinnecker, H. 1993, A\&A 278, 81

Simon, M., Ghez, A.M., Leinert, Ch. 1993, ApJ 408, L33

Walter, F.M., Vrba, J.J., Mathieu, R.D, Brown, A., Myers, P.C. 1994, AJ 107, 692 
Wichmann, R., Bastian, U., Krautter, J., Jankovics, I., Ruciński, S.M. 1998, MNRAS Letters, in press

de Zeeuw, P.T., et al. 1998 Structure and Evolution of nearby OB Associations in "HIPPARCOS Venice'97", ESA SP-402

Zinnecker, H., Brandner, W., Reipurth, B. 1992 Premain-sequence Binaries, in "Complementary Approaches to Double and Multiple Star Research", eds. H.A. McAlister \& W.I. Hartkopf, ASP Conf. Ser. 32,50

This 2-column preprint was prepared with the AAS LATEX macros v4.0. 